\title{
Pattern Formation in Epidemic Model with Media Coverage
}

\author{
Ronobir Chandra Sarker ${ }^{1} \cdot$ Saroj Kumar Sahani ${ }^{1}$ iD
}

Accepted: 27 January 2022

(c) Foundation for Scientific Research and Technological Innovation 2022

\begin{abstract}
In this article, a spatial epidemic model with media coverage is studied. By both mathematical analysis and numerical simulation, we found that there are some typical dynamics of population density such as the formation of the hole, stripe, spot, coexistence of hole and stripe or spot and stripe. The obtained results exhibit that parameters describing media coverage have a significant influence on the spatial pattern of the disease. More specifically, the sequential change in behaviour of the spatial pattern is observed as the parameter that accounts for media coverage increases. These changes analogous to the effect due to the rise in the basic reproduction number. The results presented in this article thus can be applied to any particular disease with some modification to study the effects of media coverage on disease dynamics. The model is although simple but the idea can be well extended to other complex problems.
\end{abstract}

Keywords Bifurcation $\cdot$ Media coverage $\cdot$ SI epidemic model $\cdot$ Turing pattern

Mathematics Subject Classification 35K57 · 92B05 · 35B32

\section{Introduction}

Around 12,000 years ago, when our ancestors left their nomadic hunter-gatherer society and formed the agricultural society, along with other consequences, they brought epidemics caused by viruses, and we are still fighting against the epidemic. Despite the recent development of science and technology, the epidemic diseases are still a threat to human's health. To control the epidemic, we need to understand the dynamics of diseases.

The classical models describing the dynamics of infectious diseases involves interaction between susceptible and infective. However, there are numerous environmental factors,

Ronobir Chandra Sarker is supported by "SAARC Silver Jubilee Scholarship" from South Asian University, New Delhi, India to carry out this research.

Saroj Kumar Sahani

sarojkumar@sau.ac.in

Ronobir Chandra Sarker

ronobir.sarker@gmail.com

1 Faculty of Mathematics and Computer Science, South Asian University, Akbar Bhawan Chankyapuri, New Delhi 110021, India 
such as vaccination, media coverage, migration of population etc. which can also regulate the spread of infectious diseases and can play a vital role in preventing the epidemic. Due to the recent progress of information technology, media has been used effectively as a disease control method for many infectious diseases such as H1N1, HIV/AIDS, SARS, EVD. The WHO has indicated the active role of media by rapid sharing of information in controlling the spread of H7N9 in China in 2013 [24]. The local outbreak of Zika virus in Latin America did not evolve into a global epidemic as it came into the comprehensive public view through mass media in 2016. The media such as TV News, Radio, Newspapers etc. aware the public about hazard caused from the infectious diseases and also inform them about valuable pieces of information like vaccination, avoidance of congregated places, wearing protective masks. Therefore the effective use of media may reduce the severity of a disease outbreak $[3,11,12,18]$.

The external factors like lifestyle, media coverage and density of population may affect the incidence rate. Yongfend and Jingan [9] suggested an SIS epidemic model incorporating media coverage by considering a general nonlinear incidence rate $\left(\beta_{1}-\beta_{2} \frac{I}{m+I}\right) S I$ and showed some qualitative changes due to media coverage. However, the modification of incidence rate using mass action law is not realistic for a large population. So in 2008, Jingan formulated and analysed an SIS model by considering the nonlinear incidence rate $\left(\beta_{1}-\beta_{2} \frac{I}{I+\beta_{3}}\right) \frac{S I}{S+I}$ from the modification of standard incidence rate [3].

Though the media coverage can affect the population dynamics globally, in reality, the whole population does not become aware in a short time range. As the spread of the disease dynamics happens locally, so we cannot ignore the spatial movement of the population. Many studies [4-8, 13, 20] show that spatial epidemic model is an inevitable tool in understanding complex epidemic dynamics.

Along with those stated studies, there are some studies motivated by the pioneering work of Turing [19]. Turing's revolutionary idea, which states that a stable system in the absence of diffusion can be unstable in the presence of diffusion and steady spatial pattern can emerge from this destabilisation of the symmetry is very fundamental fact to be analysed in such types of models. In 2006, Liu considered a spatial SIS model with linear incidence rate and observed some stripe patterns but no spot pattern [10]. In 2007, Sun found spot patterns along with strip patterns and coexistence of spot and stripe patterns by considering nonlinear incidence rate $\beta S^{p} I^{q}[15,17]$. In 2011, Cai investigated a spatial SIR model with a nonlinear rate of incidence of saturated mass action and found the spot, strip, stripe-spot, hole, stripe hole pattern [2]. In 2012, Wang investigated the dynamics of a spatial SI model by considering standard incidence rate and logistic growth and assuming no vertical transmission [22] and found all types of patterns as in [2]. For the interested reader, we suggest the review paper [16] for more appropriate works in these directions.

Considering all the above studies, in the present article, we propose to consider a spatial-temporal diffusive model by incorporating the incidence rate as in [3]. Using some mathematical techniques, we will investigate as to how media coverage affects the qualitative characteristics of spatial patterns in two dimensions in light of the Turing theoretical model [19]. 


\section{The Model and Mathematical Analysis}

\section{Non-spatial Model}

Let us consider total population $(P)$ is divided into two groups, the susceptible $(S)$ and the infectious $(I)$, i.e., $P=S+I$. In [1], Berezovsky proposed a model delineating the interactions between the state variables as follows:

$$
\begin{aligned}
& \frac{d S}{d t}=r P\left(1-\frac{P}{K}\right)-\mu S-\beta \frac{S I}{S+I} \\
& \frac{d I}{d t}=\beta \frac{S I}{S+I}-(\mu+v) I,
\end{aligned}
$$

where $r$ is per-capita intrinsic growth rate, $K$ is the carrying capacity, $\beta$ the infection rate constant, $\mu$ the per-capita natural mortality and $v$ the per capita disease-induced mortality.

Although in many models, the contact transmission coefficient $\beta$ was considered as a constant quantity, it can be varied for external stimulation that hinders the effective contact with the infectious population. It is ascertained in the course of the SARS outbreak in 2003 [11] that media coverage lessens contact rate and the decreasing rate depends on the number of infected individuals. So we replace the contact rate $\beta$ as some function of the number of infected individuals [3] as $\beta(I)=\beta_{1}-\beta_{2} f(I)$, where $\beta_{1}$ and $\beta_{2}$ are positive constants. Here $\beta_{1}$ can be interpreted as the contact rate without any external stimulation and $\beta_{2}$ can be considered as the maximum possible reduction from the usual contact rate through media coverage. So the model which incorporates media coverage can be assumed to be described by

$$
\begin{aligned}
& \frac{d S}{d t}=r P\left(1-\frac{P}{K}\right)-\mu S-\left(\beta_{1}-\beta_{2} \frac{I}{I+\beta_{3}}\right) \frac{S I}{S+I} \\
& \frac{d I}{d t}=\left(\beta_{1}-\beta_{2} \frac{I}{I+\beta_{3}}\right) \frac{S I}{S+I}-(\mu+v) I
\end{aligned}
$$

Normalising the model (2) through the transformations $S \rightarrow S K, I \rightarrow I K, t \rightarrow(\mu+v) t$ yields the following model

$$
\begin{aligned}
& \frac{d S}{d t}=d R_{d}(S+I)(1-(S+I))-d S-\left(R_{0}-\eta \frac{I}{I+f}\right) \frac{S I}{S+I} \\
& \frac{d I}{d t}=\left(R_{0}-\eta \frac{I}{I+f}\right) \frac{S I}{S+I}-I
\end{aligned}
$$

where $d=\frac{\mu}{\mu+v}, \eta=\frac{\beta_{2}}{\mu+v}, R_{d}=\frac{r}{\mu}, f=\frac{\beta_{3}}{K}, R_{0}=\frac{\beta}{\mu+v}$.

\section{Spatial Model}

By assuming that the susceptible (S) and infectious (I) population move randomly without affecting each other's motion (no cross-diffusion, [23]), we can obtain a simple spatial model as follows: 


$$
\begin{aligned}
& \frac{\partial S}{\partial t}=d R_{d} P(1-P)-d S-\left(R_{0}-\eta \frac{I}{I+f}\right) \frac{S I}{S+I}+D_{1} \nabla^{2} S \\
& \frac{\partial I}{\partial t}=\left(R_{0}-\eta \frac{I}{I+f}\right) \frac{S I}{S+I}-I+D_{2} \nabla^{2} I
\end{aligned}
$$

Here, the positive constants $D_{1}$ and $D_{2}$ are the diffusion coefficients of $S$ and $I$, respectively . To analyse the model (4), we consider the following non-zero initial conditions

$$
S(\mathbf{r}) \geq 0, I(\mathbf{r}, 0) \geq 0, \mathbf{r}=(x, y) \in \Omega=[0, L] \times[0, L]
$$

and zero-flux boundary conditions:

$$
\frac{\partial S}{\partial n}=\frac{\partial I}{\partial n}=0
$$

where, $n$ is the unit outward normal vector to the boundary $\partial \Omega$ of the spatial region $\Omega$.

\section{Analysis of Non-spatial Model}

Theorem 1 A The set $J=\left\{(S, I) \in \mathbb{R}_{2}^{+}: 0 \leq S+I \leq 1-\frac{\eta}{d R_{d}}\right\}$, is a positive invariant set for the dynamical system (3) if $\eta<\min \left\{R_{d}, d, 1\right\}$.

Existence of equilibrium points We can easily show that model (3) has the following types of equilibrium points, (i)The disease free non-negative equilibrium $E_{0}=\left(S_{0}, I_{0}\right)=\left(1-\frac{1}{R_{d}}, 0\right)$ for $R_{d}>1$ and (ii) an endemic equilibrium $\left(S_{e}, I_{e}\right)$ given implicitly as follows:

$$
\begin{aligned}
& c_{3} I_{e}^{3}+c_{2} I_{e}^{2}+c_{1} I_{e}+c_{0}=0 \\
& S_{e}=\frac{\left(I_{e}+f\right) I_{e}}{R_{0} I_{e}+R_{0} f-\eta I_{e}-I_{e}-f}
\end{aligned}
$$

where,

$$
\begin{aligned}
c_{0}= & d f^{2} R_{0}-d R_{d} R_{0}^{2} f^{2}+f^{2}+R_{0}^{2} f^{2}-2 R_{0} f^{2}-d f^{2}+d R_{d} R_{0} f^{2} \\
c_{1}= & -d f \eta+2 d R_{d} R_{0} f \eta+2 \eta f-\eta d R_{d} f-2 R_{0} f \eta+2 f+2 d R_{0} f \\
& -2 d R_{d} R_{0}^{2} f-4 R_{0} f+2 R_{0}^{2} f-2 d f+2 d R_{d} R_{0} f+d R_{d} R_{0}^{2} f^{2} \\
c_{2}= & \eta^{2}-d R_{d} \eta^{2}-d \eta+2 d R_{d} R_{0} \eta+2 \eta-\eta d R_{d}-2 R_{0} \eta-2 d R_{d} R_{0} f \eta \\
& +d R_{0}+1-2 R_{0}-d R_{d} R_{0}^{2}+2 d R_{d} R_{0}^{2} f+R_{0}^{2}-d+d R_{d} R_{0} \\
c_{3}= & d R_{d} \eta^{2}-2 d R_{d} R_{0} \eta+d R_{d} R_{0}^{2} .
\end{aligned}
$$

It should be noted that for $\eta=0$ the system (3) has unique positive endemic solution [22] if $R_{d}>\frac{1}{R_{0}}, R_{d}>\frac{R_{0}+d-1}{R_{0} d}$ and $R_{0}>1$.

Theorem 2 The system of equations given by (7) which describe endemic equilibrium, will have exactly one positive solution if the following conditions are satisfied: 
1. $R_{d}>\max \left\{R_{d_{1}}, R_{d_{2}}, R_{d_{3}}\right\}$

2. $1<R_{0}<2,0<\eta<1, f \geq 1$

Here,

$$
\begin{aligned}
R_{d_{1}} & =\frac{-\eta^{2}+d \eta-2 \eta+2 R_{0} \eta-d R_{0}-1+2 R_{0}-R_{0}^{2}+d}{d\left(-\eta^{2}+2 R_{0} \eta-\eta-2 R_{0} f \eta-R_{0}^{2}+2 R_{0}^{2} f+R_{0}\right)} \\
R_{d_{2}} & =\frac{d \eta-2 \eta+2 R_{0} \eta-2-2 d R_{0}+4 R_{0}-2 R_{0}^{2}+2 d}{2 d R_{0} \eta-\eta d-2 d R_{0}^{2}+2 d R_{0}+d R_{0}^{2} f} \\
R_{d_{3}} & =\frac{-d R_{0}-1-R_{0}^{2}+2 R_{0}+d}{-d R_{0}^{2}+d R_{0}}
\end{aligned}
$$

The Jacobian matrix of model (3) corresponding to this equilibrium points is

$$
A=\left(\begin{array}{ll}
a_{11} & a_{12} \\
a_{21} & a_{22}
\end{array}\right)
$$

where

$$
\begin{aligned}
a_{11}= & d R_{d}\left(1-2 S_{e}-2 I_{e}\right)-d-\left(R_{0}-\eta \frac{I_{e}}{I_{e}+f}\right) \frac{I_{e}^{2}}{\left(S_{e}+I_{e}\right)^{2}}, \\
a_{12}= & d R_{d}\left(1-2 S_{e}-2 I_{e}\right)-\left(R_{0}-\eta \frac{I_{e}}{I_{e}+f}\right) \frac{S_{e}^{2}}{\left(S_{e}+I_{e}\right)^{2}} \\
& +\frac{\eta f}{\left(I_{e}+f\right)^{2}} \frac{S_{e} I_{e}}{S_{e}+I_{e}}, \\
a_{21}= & \left(R_{0}-\eta \frac{I_{e}}{I_{e}+f}\right) \frac{I_{e}^{2}}{\left(S_{e}+I_{e}\right)^{2}}, \\
a_{22}= & \left(R_{0}-\eta \frac{I_{e}}{I_{e}+f}\right) \frac{S_{e}^{2}}{\left(S_{e}+I_{e}\right)^{2}}-\frac{\eta f}{\left(I_{e}+f\right)^{2}} \frac{S_{e} I_{e}}{S_{e}+I_{e}}-1 .
\end{aligned}
$$

As $\left(S_{e}, I_{e}\right)$ is endemic equilibrium, from 2 nd equation of (3), we get

$$
R_{0}-\eta \frac{I_{e}}{I_{e}+f}=\frac{S_{e}+I_{e}}{S_{e}} .
$$

Using (9) in (8), we get

$$
\begin{aligned}
& a_{11}=d R_{d}\left(1-2 S_{e}-2 I_{e}\right)-d-\frac{I_{e}^{2}}{S_{e}\left(S_{e}+I_{e}\right)} \\
& a_{12}=d R_{d}\left(1-2 S_{e}-2 I_{e}\right)-\frac{S_{e}}{S_{e}+I_{e}}+\frac{\eta f}{\left(I_{e}+f\right)^{2}} \frac{S_{e} I_{e}}{S_{e}+I_{e}} \\
& a_{21}=\frac{I_{e}^{2}}{S_{e}\left(S_{e}+I_{e}\right)} \\
& a_{22}=-\frac{I_{e}}{S_{e}+I_{e}}-\frac{\eta f}{\left(I_{e}+f\right)^{2}} \frac{S_{e} I_{e}}{S_{e}+I_{e}}
\end{aligned}
$$




\section{Turing Pattern Analysis}

Turing's surprising discovery [19] is that under certain conditions a spatially uniform state of a system which is stable in the absence of diffusion can be unstable in the presence of diffusion. This instability has special property of growing at a maximum finite wave length in the vicinity of equilibrium state, which in most cases can later be bounded for non-linear reaction effect of the system yielding a steady equilibrium state. As the system grows with a maximum finite wave length, so overtime that wave length dominates the final steady state with a regular pattern.

To find the conditions for Turing instability, we need to analysis the stability of (4) homogeneous equilibrium points under small perturbation. So first we write the system (4) as follows:

$$
\begin{aligned}
& \frac{\partial S}{\partial t}=f(S, I)+D_{1} \nabla^{2} S \\
& \frac{\partial I}{\partial t}=g(S, I)+D_{2} \nabla^{2} I .
\end{aligned}
$$

For this system, we are interested in the positive endemic equilibrium $E_{e}=\left(S_{e}, I_{e}\right)$. To ease our analysis, we consider that perturbation is done for specific wave vector $\mathbf{k}$. Then the non-uniform perturbations from equilibrium $E_{e}$ can be given as [16]

$$
\left(\begin{array}{l}
S \\
I
\end{array}\right)=\left(\begin{array}{c}
S_{e} \\
I_{e}
\end{array}\right)+\epsilon\left(\begin{array}{c}
S_{k} \\
I_{k}
\end{array}\right) e^{\lambda t i \mathbf{k r}}+\mathcal{O}\left(\epsilon^{2}\right),
$$

where $\lambda$ is the growth rate of perturbations over time $(t), \mathbf{r}$ is a two-dimensional radius vector. Substituting Eq. (12) in system (11) and ignoring the higher order terms of $\epsilon$, we get the stability of the system (11) depending on the eigenvalues of the following matrix:

$$
A_{k}=A-k^{2} D=\left(\begin{array}{cc}
a_{11}-k^{2} D_{1} & a_{12} \\
a_{21} & a_{22}-k^{2} D_{2} .
\end{array}\right)
$$

with $a_{i j}(i, j=1,2)$ being the elements of the Jacobian matrix corresponding to the endemic equilibrium $E_{e}$. The stability of the reduced system depends on the eigenvalues $\lambda$ 's of $A_{k}$ which is given by following equation

$$
\begin{aligned}
\lambda^{2} & +\left(\left(D_{1}+D_{2}\right) k^{2}-\left(a_{11}+a_{22}\right)\right) \lambda \\
& +\left(D_{1} D_{2} k^{4}-\left(D_{2} a_{11}+D_{1} a_{22}\right) k^{2}+a_{11} a_{22}-a_{12} a_{21}\right)=0 .
\end{aligned}
$$

For Turing instabilities, system must be stable (that means all eigenvalues will be negative) for all wave vectors $\mathbf{k}$ in the absence of diffusion. But in the absence of diffusion, (14) becomes

$$
\lambda^{2}-\left(a_{11}+a_{22}\right) \lambda+\left(a_{11} a_{22}-a_{12} a_{21}\right)=0 .
$$

From Routh-Hurwitz Theorem, conditions for which both roots of Eq. (15) will have negative real part are as follows

$$
\begin{array}{r}
a_{11}+a_{22}<0 \\
a_{11} a_{22}-a_{12} a_{21}>0
\end{array}
$$


Using (10) in (16), we have

$$
d R_{d}\left(1-2 S_{e}-2 I_{e}\right)-d-\frac{I_{e}^{2}}{S_{e}\left(S_{e}+I_{e}\right)}-\frac{I_{e}}{S_{e}+I_{e}}-\frac{\eta f}{\left(I_{e}+f\right)^{2}} \frac{S_{e} I_{e}}{S_{e}+I_{e}}<0 .
$$

and

$$
\begin{gathered}
\left(d R_{d}\left(1-2 S_{e}-2 I_{e}\right)-d-\frac{I_{e}^{2}}{S_{e}\left(S_{e}+I_{e}\right)}\right)\left(-\frac{I_{e}}{S_{e}+I_{e}}-\frac{\eta f}{\left(I_{e}+f\right)^{2}} \frac{S_{e} I_{e}}{S_{e}+I_{e}}\right) \\
-\left(d R_{d}\left(1-2 S_{e}-2 I_{e}\right)-\frac{S_{e}}{S_{e}+I_{e}}+\frac{\eta f}{\left(I_{e}+f\right)^{2}} \frac{S_{e} I_{e}}{S_{e}+I_{e}}\right)\left(\frac{I_{e}^{2}}{S_{e}\left(S_{e}+I_{e}\right)}\right)>0
\end{gathered}
$$

Turing instabilities will occur if in the presence of diffusion, at least one eigenvalue of $A_{k}$ crosses imaginary axis. So, using Routh-Hurwitz stability criterion in Eq. (14), we get Turing instability will occur if at least one of the following relation is violated

$$
\begin{array}{r}
\left(a_{11}+a_{22}\right)-\left(D_{1}+D_{2}\right) k^{2}<0, \\
D_{1} D_{2} k^{4}-\left(D_{2} a_{11}+D_{1} a_{22}\right) k^{2}+a_{11} a_{22}-a_{12} a_{21}>0 .
\end{array}
$$

Since $a_{11}+a_{22}<0, D_{1}, D_{2}>0$, and $k^{2}>0$, clearly 1 st equation of (19) cannot be violated. So Turing instability can occur if 2 nd equation of (19) is violated. So we have

$$
D_{1} D_{2} k^{4}-\left(D_{2} a_{11}+D_{1} a_{22}\right) k^{2}+a_{11} a_{22}-a_{12} a_{21}<0 .
$$

Left hand side of (20) can be considered as a quadratic polynomial in $k^{2}$, so (20) will be true if minimum value of that polynomial becomes negative. It can be easily shown that the minimum occurs at the wave vector $\mathbf{k}$ given by

$$
k^{2}=\frac{D_{1} a_{22}+D_{2} a_{11}}{2 D_{1} D_{2}} .
$$

The corresponding minimum value is

$$
a_{11} a_{22}-a_{12} a_{21}-\frac{\left(D_{1} a_{22}+D_{2} a_{11}\right)^{2}}{4 D_{1} D_{2}} .
$$

Instability will occur if this expression is negative, so we have

$$
D_{1} a_{22}+D_{2} a_{11}>2 \sqrt{D_{1} D_{2}\left(a_{11} a_{22}-a_{12} a_{21}\right)} \text {. }
$$

As $k^{2}$ cannot be negative, so from (21), we have

$$
D_{1} a_{22}+D_{2} a_{11}>0
$$

From Eq. (20), it can be shown that when this transition from stability to instability occurs, $k^{2}$ ranges between the critical values $k_{-}$and $k_{+}$where

$$
k_{ \pm}^{2}=\frac{a_{11} D_{2}+a_{22} D_{1}-\sqrt{\left(a_{11} D_{2}+a_{22} D_{1}\right)^{2}-4 D_{1} D_{2}\left(a_{11} a_{22}-a_{12} a_{21}\right)}}{2 D_{1} D_{2}}
$$




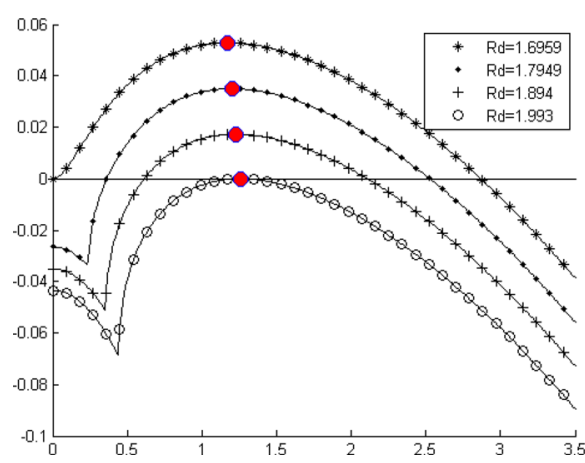

(a) $\eta=0.3$

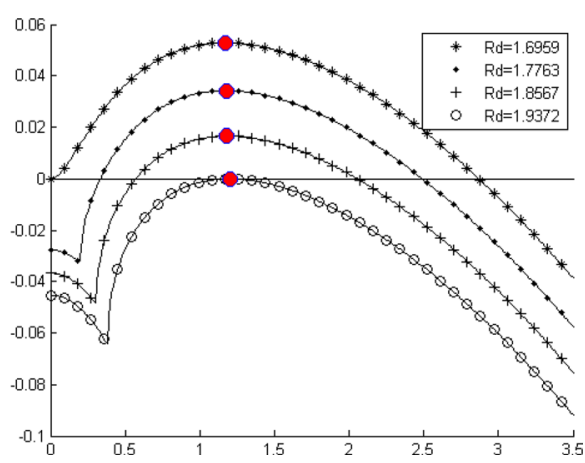

(b) $\eta=0.9$

Fig. 1 Basic dispersion relation giving the graph of excursion rate $\operatorname{Re}(\lambda)$ vs wavenumber $|k|$ with $R_{0}=1.14, d=0.15, f=1, D_{1}=0.01, D_{2}=0.25$ for different value of $\eta$

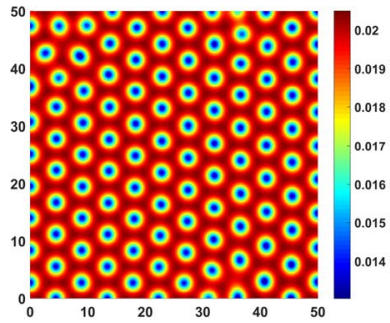

(a) $R_{0}=1.140$

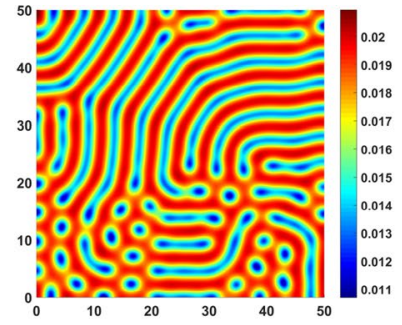

(b) $R_{0}=1.154$

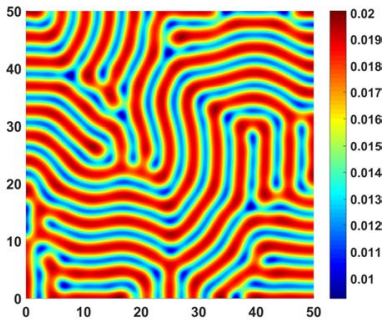

(c) $R_{0}=1.170$

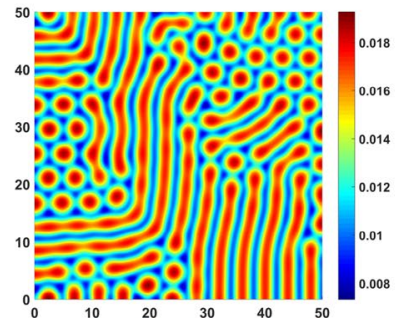

(d) $R_{0}=1.200$

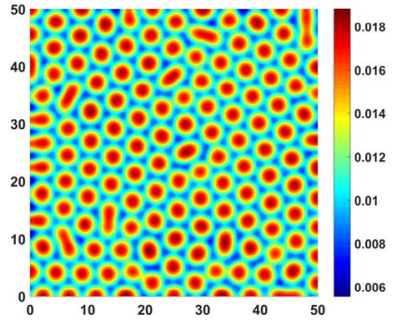

(e) $R_{0}=1.213$

Fig. 2 The five categories of Turing patterns of $I$ in model (4) with parameters $R_{d}=2, d=0.15, f=1$, $\eta=0, D_{1}=0.01, D_{2}=0.25$. a $R_{0}=1.14$. b $R_{0}=1.154$. $\mathbf{c} R_{0}=1.17$. d $R_{0}=1.2$. e $R_{0}=1.213$

Condition (23) ensures the existence of this range of $k^{2}$ such that $k_{-}^{2}<k^{2}<k_{+}^{2}$ which will grow over the time and (24) ensures that lower limit will be greater than zero so that $0<k_{-}^{2}<k^{2}<k_{+}^{2}$. There is also a critical value $k_{c}$ within that range at which excursions are fastest growing.

In the following section, we have simulated the model for specific set of parameter to look for some pattern as the time evolves. We first plotted Figs. 1 and 2 to start our exhaustive simulations on the system (4).

Figure 1 shows the growth rate vs wavenumber $|\mathbf{k}|$. We considered $\eta=0.3,0.9$ successively for each figures in Fig. 1. In each of those figures, we have plotted the growth vs 
wavenumber for different $R_{d}$ where other parameters were kept fixed. We observe that for each $\eta$, there exists some minimum $R_{d}$ below which lower bound of excursive wavenumber goes to negative. So for this $R_{d}$ value, an excursion at 0 wavenumber (i.e. infinite wavelength) occurs. Also there exists some maximum $R_{d}$ beyond which there is no excursion for any wavenumber. For values $R_{d}$ which lies between those minimum and maximum values, excursion yielding wavenumbers lie within a range of positive numbers. So wavelength also lies between a finite range. Since wavelength is in a finite length, some regular patterns are formed which are called Turing pattern. We also observe that upper limit of $R_{d}$ decreases as $\eta$ increases.

In the following section, we have numerically simulated the model to look out how different patterns can be formed as the time evolves. We have applied a simple finite difference technique which is described below.

\section{Turing Pattern Formation}

\section{Numerical Technique}

Though it is possible to find analytically the parameter sets for which Turing pattern will be formed, there is no general method to forecast the final pattern for a specific set of parameter values. So we need to carry out numerical simulations of the proposed model (4). All of our numerical simulations employ non-zero initial condition and zero-flux boundary conditions and part of the parameter values are chosen according to [22]. The continuous problem is transformed to a discrete problem by considering a discrete domain with $M \times N$ lattice points with $h$ spacing and discrete time step with step size $\tau$. The 2D Laplacian $\Delta$ is discretised using the standard five-point approximation and time derivative is discretised using Euler's method. Eventually, the concentrations $\left(S_{i, j}^{n+1}, I_{i, j}^{n+1}\right)$ at the discrete moment $(n+1) \tau$ at some lattice point $\left(x_{i}, y_{j}\right)$ are given by

$$
\begin{aligned}
& S_{i, j}^{n+1}=S_{i, j}^{n}+\tau D_{1} \Delta_{h} S_{i, j}^{n}+\tau f\left(S_{i, j}^{n}, I_{i, j}^{n}\right), \\
& I_{i, j}^{n+1}=I_{i, j}^{n}+\tau D_{2} \Delta_{h} I_{i, j}^{n}+\tau g\left(S_{i, j}^{n}, I_{i, j}^{n}\right),
\end{aligned}
$$

with the Laplacian defined by

$$
\Delta_{h} S_{i, j}^{n}=\frac{S_{i+1, j}^{n}+S_{i-1, j}^{n}+S_{i, j-1}^{n}-4 S_{i, j}^{n}}{h^{2}} .
$$

We consider a initial state which is slightly perturbed from endemic equilibrium state $\left(S_{e}, I_{e}\right)$ and consider parameter values which are in Turing space. Then as the system is integrated, the system generally tends to a steady state (time independent) solution. We have run our simulation till no essential change in state happens i.e. a steady state is attained. We have show only distributions of infectious population $I$ in every simulations.

\section{Simulation}

Variation of disease pattern for different $R_{0}$ The behaviour of the system with respect to parameter $R_{0}$ is shown in Fig. 2 which depicts the changes of final inhomogeneous steady 
state as $R_{0}$ increases. We observe that the pattern sequence follows hole $\rightarrow$ stripe-hole $\rightarrow$ stripe $\rightarrow$ stripe-spot $\rightarrow$ spot.

Variation of disease pattern for different $\eta$ Figure 3 shows the Turing patterns for different $\eta$ considering other parameters as $R_{d}=2, d=0.15, f=1, R_{0}=1.154, D_{1}=0.01$, $D_{2}=0.25$. One can see that when $\eta$ varies from 0 to 0.5 the pattern now follows the sequence stripe-holes $\rightarrow$ holes. On the other hand, in Fig. 4, we observe the sequence now follows stripes $\rightarrow$ stripe-holes $\rightarrow$ holes. The sequences in Figs. 5, 6 are respectively "Stripespots $\rightarrow$ stripes" and "Spots $\rightarrow$ stripe-spots $\rightarrow$ stripes". So we observe that for increasing values of $\eta$, sequence of patterns is Spots $\rightarrow$ stripe-spots $\rightarrow$ stripes $\rightarrow$ stripe-holes $\rightarrow$ holes which is opposite to the sequence of patterns for increasing values of $R_{0}$.

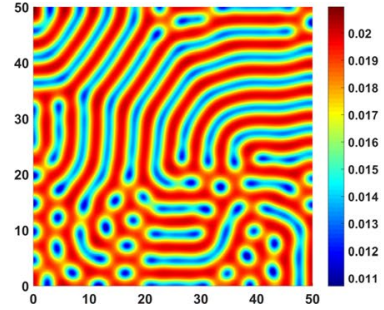

(a) $\eta=0$

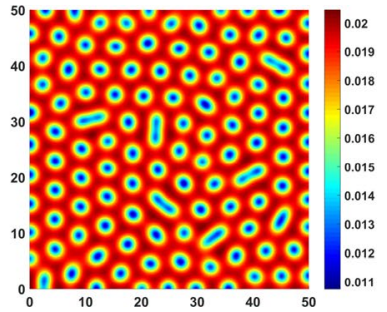

(b) $\eta=0.3$

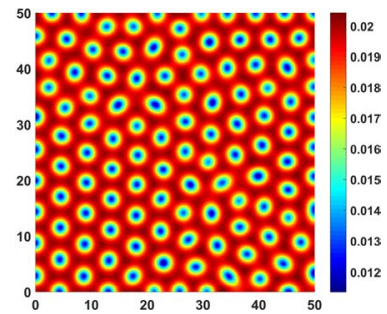

(c) $\eta=0.5$

Fig. 3 Turing patterns of $I$ in model (4) with parameters $R_{d}=2, d=0.15, f=1, R_{0}=1.154, D_{1}=0.01$, $D_{2}=0.25$. $\mathbf{a} \eta=0$. $\mathbf{b} \eta=0.3$. $\mathbf{c} \eta=0.5$

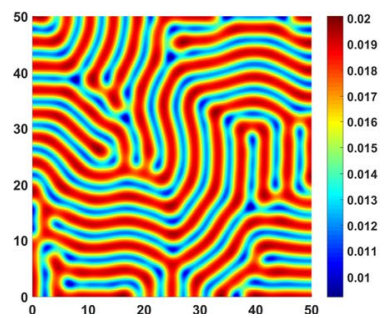

(a) $\eta=0$

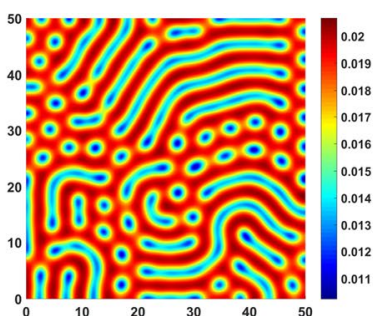

(b) $\eta=1$

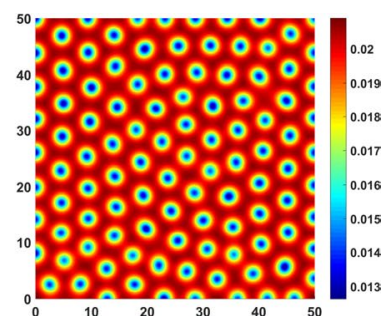

(c) $\eta=1.7$

Fig. 4 Turing patterns of $I$ in model (4) with parameters $R_{d}=2, d=0.15, f=1, R_{0}=1.17, D_{1}=0.01$, $D_{2}=0.25$. a $\eta=0 . \mathbf{b} \eta=1$. $~ \eta=1.7$

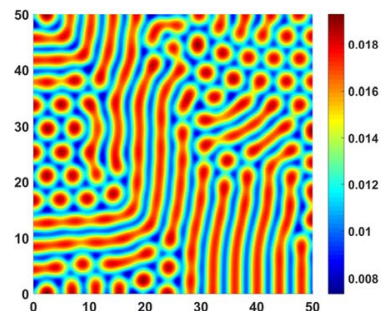

(a) $\eta=0$

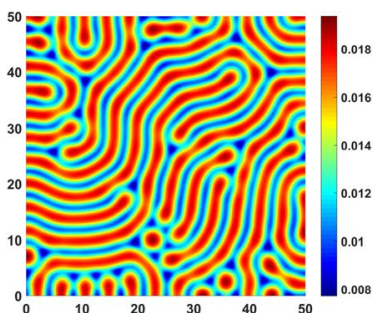

(b) $\eta=0.5$

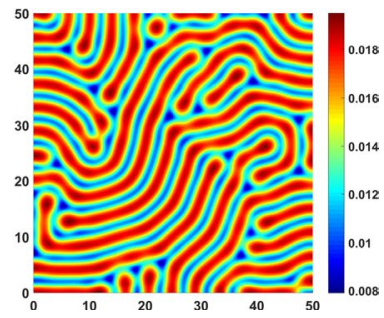

(c) $\eta=1.0$

Fig. 5 Turing patterns of $I$ in model (4) with parameters $R_{d}=2, d=0.15, f=1, R_{0}=1.2, D_{1}=0.01$, $D_{2}=0.25$. a $\eta=0 . \mathbf{b} \eta=0.5 . \mathbf{c} \eta=1$ 


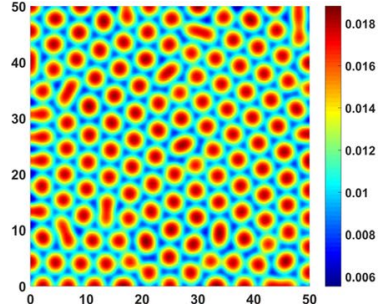

(a) $\eta=0$

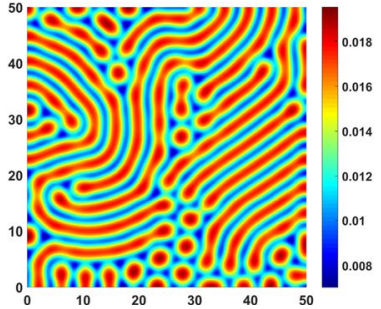

(b) $\eta=1$

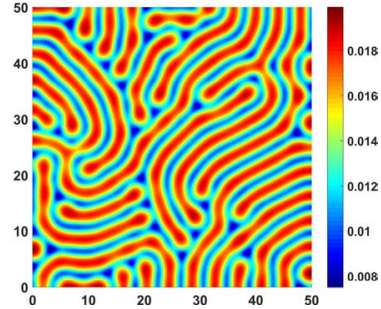

(c) $\eta=1.5$

Fig. 6 Turing patterns of $I$ in model (4) with parameters $R_{d}=2, d=0.15, f=1, R_{0}=1.213, D_{1}=0.01$, $D_{2}=0.25 . \mathbf{a} \eta=0 . \mathbf{b} \eta=1$. $\mathbf{c} \eta=1.5$

\section{Conclusion and Remarks}

The epidemic model with media coverage are investigated by several researchers $[9,14,21$, 25]. However to the best of our knowledge, spatial distribution of the population has not been taken into account in the literature. This motivate us to consider spatial distribution of population and investigate the effect of media coverage i.e. $\eta$ parameter in formation of spatial pattern. We have analysed the model analytically and simulated the models numerically to determine the long term dynamics of the disease spread into the population. From the above results, we observed that the effect of $\eta$ term on formation of spatial pattern is opposite to the effect of basic reproduction number $R_{0}$ on the formation of spatial pattern. As $R_{0}$ being increased, pattern dynamics sequence is hole $\rightarrow$ hole-stripe $\rightarrow$ stripe $\rightarrow$ spot-stripe $\rightarrow$ spot. As $\eta$ is increased, pattern dynamics sequence then follows spot $\rightarrow$ spot-stripe $\rightarrow$ stripe $\rightarrow$ holestripe $\rightarrow$ hole. So for a fixed $R_{0}$ value, we can control spatial dynamics by simply controlling the value of $\eta$. Moreover changes of $\eta$ yields qualitative change for the long term behaviour of disease spread. These a priori information can help us to detect where diseases are more pervasive and thereby can help us to optimise the cost of media coverage. More exhaustive studies are necessary to reveal impact of media coverage more accurately by incorporating more complex spatial epidemic models like SEIRS, SIRS and others. We therefore propose to model such complex dynamics in our future articles.

\section{Proof of Theorem 1}

Proof By examining the evolution of solutions along time on the boundary of $J$, we can verify that $J$ is positive invariant. First let us consider the boundary $J_{b 1}=\left\{(S, 0): 0 \leq S \leq 1-\frac{\eta}{d R_{d}}\right\}$. On this boundary $J_{b 1}$ the dynamical system (3) takes the form

$$
\begin{aligned}
& \frac{d S}{d t}=d R_{d} S(1-S)-d S \\
& \frac{d I}{d t}=-I .
\end{aligned}
$$

Solution of 1 st equation of (26) is 


$$
S(t)=\frac{R_{d}-1}{R_{d}+C_{1}\left(R_{d}-1\right) e^{-v\left(R_{d}-1\right) t}} .
$$

Now if initially $S$ is non-negative i.e. $S(0) \geq 0$, then $\frac{R_{d}-1}{R_{d}+C_{1}\left(R_{d}-1\right)} \geq 0$ and if $R_{d}-1<0$, then this inequality is only true when

$$
R_{d}+C_{1}\left(R_{d}-1\right)<0,
$$

which on simplification, gives $R_{d}+C_{1}\left(R_{d}-1\right) e^{-v\left(R_{d}-1\right) t}<0$, and so from expression (27), we get $S(t)>0$. On the other hand if $R_{d}-1>0$, then inequality [24] implies

$$
R_{d}+C_{1}\left(R_{d}-1\right)>0
$$

which leads to $R_{d}+C_{1}\left(R_{d}-1\right) e^{-v\left(R_{d}-1\right) t}>0$, and therefore from expression (27), we have $S(t)>0$. Now, solution of second equation of (26) is

$$
I=I(0) e^{-t} \text {. }
$$

If initially $I_{\epsilon}$ is non-negative, then $I(t)$ is always negative. Hence any point on the boundary $J_{b 1}$ solution will evolved to be non-negative. Now let us consider the following boundary

$$
J_{b 2}=\left\{(0, I): 0 \leq I \leq 1-\frac{\eta}{d R_{d}}\right\} .
$$

On this boundary, the dynamical system (3) takes the form

$$
\begin{aligned}
& \frac{d S}{d t}=d R_{d} I(1-I) \\
& \frac{d I}{d t}=-I,
\end{aligned}
$$

and so, solution of second equation of Eq. (29) is

$$
I=I(0) e^{-t} \text {. }
$$

So it is clear that if $I$ is initially non-negative, it will be evolved to be non-negative. Again if $I$ is initially in $J$ then $I \leq 1$. So we have

$$
\frac{d S}{d t}=d R_{d} I(1-I) \geq 0
$$

implying that $S$ is non-negative. Hence any point on the boundary $J_{b 2}$, solution will be non-negative.

Finally, consider the boundary

$$
J_{b 3}=\left\{(S, I): S \geq 0, I \geq 0, S+I=1-\frac{\eta}{d R_{d}}\right\} .
$$

Summing up the two equations in (26), followed by simplification, it leads to

$$
\frac{d P}{d t} \leq\left(d R_{d}-\eta-d R_{d} P\right) P .
$$

Now for $P=1-\frac{\eta}{d R_{d}}, \frac{d P}{d t} \leq 0$, so we have $P(t) \leq 1-\frac{\eta}{v R_{d}}$ for all $t$ and this completes the proof. 


\section{Proof of Theorem 2}

Proof Assume that $R_{0}>1, \eta<1$, then

$$
c_{3}=d R_{d} \eta^{2}-2 d R_{d} R_{0} \eta+d R_{d} R_{0}^{2}=d R_{d}\left(\eta-R_{0}\right)^{2}>0 .
$$

Again,

$$
\begin{aligned}
- & \eta^{2}+2 R_{0} \eta-\eta-2 R_{0} f \eta-R_{0}^{2}+2 R_{0}^{2} f+R_{0} \\
& =\eta\left(2 R_{0}-(\eta+1)\right)+R_{0}\left(R_{0}(2 f-1)-(2 f \eta-1)\right) \\
& >\eta(2-(1+1))+R_{0}((2 f-1)-(2 f-1))=0\left(\because R_{0}>1, \eta<1\right) \\
& \Rightarrow-\eta^{2}+2 R_{0} \eta-\eta-2 R_{0} f \eta-R_{0}^{2}+2 R_{0}^{2} f+R_{0}>0
\end{aligned}
$$

So we have,

$$
\begin{aligned}
R_{d} & >\frac{-\eta^{2}+d \eta-2 \eta+2 R_{0} \eta-d R_{0}-1+2 R_{0}-R_{0}^{2}+d}{d\left(-\eta^{2}+2 R_{0} \eta-\eta-2 R_{0} f \eta-R_{0}^{2}+2 R_{0}^{2} f+R_{0}\right)} \\
& \Rightarrow c_{2}>0
\end{aligned}
$$

Again,

$$
\begin{aligned}
& 2 d R_{0} \eta-\eta d-2 d R_{0}^{2}+2 d R_{0}+d R_{0}^{2} f \\
& \quad=\eta d\left(2 R_{0}-1\right)+d R_{0}\left(-2 R_{0}+2+R_{0} f\right) \\
& \quad>\eta d(2-1)+d R_{0}(-2 \times 2+2+2 f) \\
& \quad \Rightarrow 2 d R_{0} \eta-\eta d-2 d R_{0}^{2}+2 d R_{0}+d R_{0}^{2} f>0
\end{aligned}
$$

So we have,

$$
R_{d}>\frac{d \eta-2 \eta+2 R_{0} \eta-2-2 d R_{0}+4 R_{0}-2 R_{0}^{2}+2 d}{2 d R_{0} \eta-\eta d-2 d R_{0}^{2}+2 d R_{0}+d R_{0}^{2} f} \Rightarrow c_{1}>0
$$

Again, $-d R_{0}^{2}+d R_{0}=d R_{0}\left(-R_{0}+1\right)<0\left(\because R_{0}>1\right)$

So we have,

$$
R_{d}>\frac{-d R_{0}-1-R_{0}^{2}+2 R_{0}+d}{-d R_{0}^{2}+d R_{0}} \Rightarrow c_{0}<0
$$

So from Descartes' rule of signs, we can conclude that 1st equation of (7) has exactly one positive solution. So from 2 nd equation of (7), we have

$$
S_{e}=\frac{\left(I_{e}+f\right) I_{e}}{R_{0} I_{e}+R_{0} f-\eta I_{e}-I_{e}-f}=\frac{I_{e}}{R_{0}-1-\frac{\eta I_{e}}{I_{e}+f}}
$$

Acknowledgements The authors are grateful to the esteemed reviewers for their constructive comments and suggestions. It has helped us greatly in making the manuscript better in every aspect. 


\section{References}

1. Berezovskaya, F., Karev, G., Song, B., Castillo-Chavez, C.: A simple epidemic model with surprising dynamics. Math. Biosci. Eng. 2(1), 133 (2005)

2. Cai, Y., Wang, W.: Spatiotemporal dynamics of a reaction-diffusion epidemic model with nonlinear incidence rate. J. Stat. Mech. Theory Exp. 2011(02), 02025 (2011)

3. Cui, J., Tao, X., Zhu, H., et al.: An sis infection model incorporating media coverage. Rocky Mt. J. Math. 38(5), 1323-1334 (2008)

4. Festenberg, N.V., Gross, T., Blasius, B.: Seasonal forcing drives spatio-temporal pattern formation in rabies epidemics. Math. Model. Nat. Phenom. 2(4), 63-73 (2007)

5. Funk, G.A., Jansen, V.A.A., Bonhoeffer, S., Killingback, T.: Spatial models of virus-immune dynamics. J. Theor. Biol. 233(2), 221-236 (2005)

6. Grenfell, B.T., Bjørnstad, O.N., Kappey, J.: Travelling waves and spatial hierarchies in measles epidemics. Nature 414(6865), 716-723 (2001)

7. He, D., Stone, L.: Spatio-temporal synchronization of recurrent epidemics. Proc. R. Soc. B Biol. Sci. 270(1523), 1519-1526 (2003)

8. Hosono, Y., Ilyas, B.: Traveling waves for a simple diffusive epidemic model. Math. Models Methods Appl. Sci. 05(07), 935-966 (1995)

9. Li, Y., Cui, J.: The effect of constant and pulse vaccination on SIS epidemic models incorporating media coverage. Commun. Nonlinear Sci. Numer. Simul. 14(5), 2353-2365 (2009)

10. Liu, Q.X., Jin, Z.: Formation of spatial patterns in epidemic model with constant removal rate of the infectives. J. Stat. Mech. Theory Exp. 05002, 7 (2006)

11. Liu, R., Wu, J., Zhu, H.: Media/psychological impact on multiple outbreaks of emerging infectious diseases. Comput. Math. Methods Med. 8(3), 153-164 (2007)

12. Liu, Y., Cui, J.A.: The impact of media coverage on the dynamics of infectious disease. Int. J. Biomath. 01(01), 65-74 (2008)

13. Lloyd, A.L., Jansen, V.A.A.: Spatiotemporal dynamics of epidemics: synchrony in metapopulation models. Math. Biosci. 188, 1-16 (2004)

14. Misra, A.K., Sharma, A., Shukla, J.B.: Modeling and analysis of effects of awareness programs by media on the spread of infectious diseases. Math. Comput. Model. 53(5-6), 1221-1228 (2011)

15. Sun, G., Jin, Z., Liu, Q.X., Li, L.: Pattern formation in a spatial SI model with non-linear incidence rates. J. Stat. Mech. Theory Exp. 2007(11), 11011 (2007)

16. Sun, G.Q.: Mathematical modeling of population dynamics with Allee effect. Nonlinear Dyn. 85(1), 1-12 (2016)

17. Sun, G.Q., Jin, Z., Liu, Q.X., Li, L.: Chaos induced by breakup of waves in a spatial epidemic model with nonlinear incidence rate RID F-5904-2010 RID E-4892-2010. J. Stat. Mech. Theory Exp. 08011, 0811 (2008)

18. Tchuenche, J.M., Dube, N., Bhunu, C.P., Smith, R.J., Bauch, C.T.: The impact of media coverage on the transmission dynamics of human influenza. BMC Public Health 11(Suppl 1), S5 (2011)

19. Turing, A.M.: The chemical basis of morphogenesis. Philos. Trans. R. Soc. Lond. B 237(641), 37-72 (1952)

20. Wang, K., Wang, W., Song, S.: Dynamics of an HBV model with diffusion and delay. J. Theor. Biol. 253(1), 36-44 (2008)

21. Wang, L., Zhou, D., Liu, Z., Xu, D., Zhang, X.: Media alert in an SIS epidemic model with logistic growth. J. Biol. Dyn. 11(May), 120-137 (2017)

22. Wang, W., Cai, Y., Wu, M., Wang, K., Li, Z.: Complex dynamics of a reaction-diffusion epidemic model. Nonlinear Anal. Real World Appl. 13(5), 2240-2258 (2012)

23. Wang, Y., Wang, J., Zhang, L.: Cross diffusion-induced pattern in an SI model. Appl. Math. Comput. 217(5), 1965-1970 (2010)

24. WHO: Overview of the emergence and characteristics of the avian influenza A (H7N9) virus. WHO report (May), 1-38 (2013)

25. Zhang, L., Lin, L., Ge, J.: A diffusive SIS epidemic model incorporating the media coverage impact in the heterogeneous environment. Discrete Contin. Dyn. Syst. Ser. B 22(7), 2763-2776 (2017)

Publisher's Note Springer Nature remains neutral with regard to jurisdictional claims in published maps and institutional affiliations. 